\title{
Selamento apical em obturações retrógradas. Estudo em dentes bovinos empregando solução corante Rodamina B
}

\author{
Apical sealing in retrograde fillings. Study in bovine teeth using Rhodamine B dye solution \\ Gustavo SIVIERI-ARAÚJO \\ Doutorando - Programa de Pós-Graduação em Endodontia - Departamento de Odontologia Restauradora - Faculdade \\ de Odontologia de Araraquara - Universidade Estadual Paulista - UNESP - Araraquara - SP - Brasil
}

\section{Eduardo Antunes BORTOLUZZI}

Doutorando - Programa de Pós-Graduação em Endodontia - Departamento de Odontologia Restauradora - Faculdade de Odontologia de Araraquara - Universidade Estadual Paulista - UNESP - Araraquara - SP - Brasil

\author{
Juliane Maria Guerreiro TANOMARU \\ Professora Assistente Doutora - Departamento de Odontologia Restauradora - Disciplina de Terapêutica - Faculdade \\ de Odontologia de Araraquara - Universidade Estadual Paulista - UNESP - Araraquara - SP - Brasil
}

\section{Fábio Luiz Camargo Villela BERBERT}

Professor Assistente Doutor - Departamento de Odontologia Restauradora - Disciplina de Endodontia - Faculdade de Odontologia de Araraquara - Universidade Estadual Paulista - UNESP - Araraquara - SP - Brasil

\section{Mário TANOMARU-FILHO}

Professor Adjunto - Departamento de Odontologia Restauradora - Disciplina de Endodontia - Faculdade de Odontologia de Araraquara - Universidade Estadual Paulista - UNESP - Araraquara - SP - Brasil

\begin{abstract}
Resumo
A capacidade de selamento de materiais utilizados em endodontia tem sido avaliada por testes de infiltração com corantes. A proposta deste estudo foi avaliar o selamento apical de materiais utilizados em obturações retrógradas empregando modelo experimental com dentes bovinos. Quarenta incisivos bovinos recém extraídos, com raízes retas tiveram seus canais radiculares instrumentados e obturados. Após a secção da porção apical, cavidades retrógradas foram preparadas com pontas de retropreparo ultra-sônico e os dentes divididos aleatoriamente em três grupos experimentais $(n=12)$ e quatro controles $(n=4)$. Foram utilizados os materiais: Grupo I - MTA Angelus Cinza; Grupo II - Cimento de Óxido Zinco e Eugenol; Grupo III - Sealer 26. Em seguida, os dentes foram imersos em solução de Rodamina B a 0,2 \% por 48 horas em ambiente com vácuo. Decorrido este período, os dentes foram seccionados longitudinalmente e as imagens digitalizadas analisadas por meio do programa Image Tool. Grupos controle positivo e negativo apresentaram infiltração máxima ou zero, respectivamente. Os resultados obtidos foram submetidos ao teste ANOVA e de Tukey demonstrando menor infiltração apical para o Sealer $26(\mathrm{p}<0,05)$. MTA e OZE apresentaram infiltração apical semelhante $(\mathrm{p}>0,05)$. Conclui-se que o cimento Sealer 26 proporciona selamento apical superior ao MTA e OZE, sendo o modelo experimental com dentes bovinos viável para a avaliação do selamento apical em obturações retrógradas.
\end{abstract}

\section{UNITERMOS}

Endodontia; infiltração dentária; material retroobturador. 


\section{INTRODUÇÃo}

A cirurgia parendodôntica constitui um recurso indicado quando há falha e/ou impossibilidade do tratamento endodôntico convencional. Dentre as modalidades cirúrgicas existentes, a obturação retrógrada consiste no preparo de cavidade apical e preenchimento com material obturador retrógrado 7 , o qual deve apresentar propriedades físico-químicas adequadas e compatibilidade biológica.

Dentre os materiais retrobturadores, o Mineral Trióxido Agregado (MTA), foi desenvolvido na Universidade de Loma Linda, na Califórnia, EUA, e citado pela primeira vez por Lee et al. ${ }^{11}$ em 1993, sendo composto de silicato tricálcico, aluminato tricálcico e outros óxidos minerais, apresentado boas propriedades para uso como material retrobturador.

O MTA apresenta capacidade seladora satisfatória como material usado na retrobturação $0^{25,27}$, bem como no selamento de perfurações radiculares ${ }^{11}$. O MTA também apresenta biocompatibilidade adequada tanto nos casos de obturações retrógradas ${ }^{26}$, como no tratamento de perfurações radiculares ${ }^{8}$.

Para avaliar a capacidade seladora de cimentos obturadores e retrobturadores, tem sido utilizado o método de infiltração marginal com soluções corantes, como por exemplo, o azul de metileno ${ }^{14,24,27}$.

Entretanto, devido à incompatibilidade do azul de metileno com materiais alcalinos, o seu uso vem sendo questionado. Esta solução pode sofrer descoloração quando em contato com substânciais alcalinas, reduzindo assim a eficácia de marcação do corante ${ }^{10,20}$. Considerando-se que o MTA apresenta propriedades alcalinas, promovendo elevação do $\mathrm{pH}^{6,20}$, o emprego da solução de azul de metileno para avaliação de sua capacidade seladora deve ser evitado. Desta forma, o emprego de soluções corantes compatíveis com materiais alcalinos tem conduzido ao emprego da solução de Rodamina B.

Devido a dificuldade de obtenção de dentes de humanos para realização de pesquisas, modelos alternativos como o dente bovino têm sido sugeridos. Nakamishi et al. ${ }^{13}$ (1983), compararam em teste de adesão, a dentina humana com a bovina, sendo observada redução da resistência adesiva na dentina bovina profunda. A avaliação por Microscopia Eletrônica de Varredura demonstrou que a dentina humana e bovina foram semelhantes antes e após o experimento. O emprego de dentes bovinos em teste de infiltração foi relatado por Wu et al. ${ }^{29}$ (1998), empregando 100 raízes bovinas a fim de verificar o selamento marginal em obturações retrógradas realizadas com amálgama, Super-EBA e MTA em longos períodos experimentais. O MTA demonstrou menor infiltração que a amálgama e Super-EBA e os autores consideraram os dentes bovinos compatíveis para testes de infiltração marginal.

Desta forma, considerando-se a dificuldade de obtenção e questões éticas relacionadas ao emprego de dentes de humanos, a sua substituição por dentes bovinos tem sido investigada. Para isto, são necessárias avaliações em dentes bovinos empregando metodologia semelhante à empregada em dentes de humanos. Com relação ao estudo da capacidade seladora em obturações retrógradas, Tanomaru-Filho et al. ${ }^{21}$ (2005), observaram, após avaliação de infiltração marginal em obturações retrógradas realizadas in vitro em dentes de humanos, que o MTA e óxido de zinco e eugenol apresentaram resultados semelhantes e inferiores ao Sealer 26.

A proposta do presente estudo foi avaliar o selamento apical proporcionado por diferentes materiais retrobturadores em teste de infiltração empregando corante Rodamina B, utilizando dentes bovinos como modelo experimental.

\section{Material e método}

Foram utilizados neste estudo, quarenta incisivos de bovinos recém extraídos, com raízes retas e íntegras, que foram estocados em solução salina a $0,9 \%$, após armazenagem em solução de hipoclorito de sódio a $2 \%$ por 48 horas.

Os espécimes foram seccionados transversalmente na junção amelo-cementária com disco de carborudum, sob refrigeração. O comprimento real do dente foi estabelecido por meio de um instrumento tipo-K 15 (Dentsply-Maillefer, Ballaigues, Suiça) posicionada no canal radicular até a abertura foraminal. O comprimento real de trabalho foi determinado $1 \mathrm{~mm}$ aquém deste comprimento, sendo o preparo biomecânico realizado com instrumentos tipo-K e irrigação com solução de hipoclorito de sódio a $1 \%$, até o instrumento K \# 80 em todos os canais radiculares. Em seguida, os canais radiculares foram secos e obturados com cones de guta-percha principal \# 80 e cones de guta-percha auxiliares $\mathrm{F}$ e MF (Tanariman Indústria Ltda., Manacapuru, AM, Brasil) e cimento à base de óxido de zinco e eugenol - Intrafill (S.S. White Artigos Dentários Ltda., Rio de Janeiro, RJ, Brasil), adaptados no comprimento real de trabalho, utilizando a técnica de obturação da condensação lateral ativa. 
Decorrido 24 horas da obturação, as raízes foram submetidas à apicectomia, usando uma broca Endo-Z (Dentsply Indústria e Comércio Ltda Petrópolis, RJ, Brasil) em alta rotação. A apicectomia foi realizada perpendicular ao longo eixo radicular, $3 \mathrm{~mm}$ aquém do ápice radicular. Cavidades retrógradas de $3 \mathrm{~mm}$ de profundidade, foram preparadas utilizando ultra-som (Enac, Osada, Japan) e pontas de ultra-som (S12D/90 - Osada, Japan), com contínua irrigação de solução salina.
Após o preparo das cavidades, as raízes foram secas e a impermeabilização da superfície radicular externa foi realizada com duas camadas de adesivos (Araldite-Ciba-Geigy S.A., Taboão da Serra, SP, Brasil) e duas camadas de esmalte de unha, exceto na superfície apical seccionada. As raízes foram divididas aleatoriamente em três grupos experimentais com doze espécimes cada, e os grupos controle com duas raízes (Quadro 1).

\section{Quadro 1 -Distribuição dos espécimes e grupos experimentais de acordo com os materiais utilizados}

\begin{tabular}{l|l|l}
\hline GRUPOS & MATERIAL RETROBTURADOR & RODAMINA B a 0,2 \% \\
\hline I & MTA Ângelus cinza* & 12 dentes \\
II & Óxido de Zinco e Eugenol ${ }^{\star \star}$ & 12 dentes \\
III & Sealer 26 & 12 dentes \\
IV & Controle positivo - sem material & 2 dentes \\
\hline V & Controle negativo - impermeabilização total & 2 dentes \\
\hline
\end{tabular}

* Angelus, Londrina, PR, Brasil

** S.S. White Artigos Dentários Ltda., Rio de Janeiro, RJ, Brasil

*** Dentsply Indústria e Comércio Ltda., Petrópolis, RJ, Brasil

As cavidades retrógradas foram preenchidas com os três materiais estudados, MTA, óxido de zinco e eugenol e Sealer 26, respectivamente. Dentre as quatro raízes do grupo controle, duas não receberam material retrobturador (controle positivo) e as outras duas restantes, tiveram as cavidades retrógradas totalmente impermeabilizadas (controle negativo) após obturação retrógrada com um dos materiais em estudo.

O óxido de zinco e eugenol (OZE) foi manipulado na proporção pó/líquido $1 \mathrm{~g} / 0,2 \mathrm{ml}$, conforme preconizado por Bernabé . O MTA Angelus cinza de acordo com as instruções do fabricante e o Sealer 26 acrescido de uma maior quantidade de pó, formando uma consistência mais espessa na proporção pó/líquido de 5:1, de acordo com Tanomaru-Filho et al. ${ }^{23}$ (2006).

Após as retrobturações os grupos experimentais foram imersos em solução de Rodamina B a $0,2 \%$, em ambiente de vácuo produzido por bomba de aspiração (Tecnal TE-058, Indústria para Laboratórios, Piracicaba, SP, Brazil). Todos os grupos foram mantidos imersos nas soluções corantes por 48 horas.

Depois da imersão nos corantes, as raízes foram lavadas em água corrente por 24 horas. A impermeabilização foi removida e as raízes foram seccionadas longitudinalmente de vestibular por lingual com um disco diamantado sob refrigeração, permitindo assim a exposição da cavidade e do material retrobturador. Em seguida, foram realizadas fotografias com a máquina fotográfica digital Sony - Cyber-shot DSCF717 (Sony Corporation, Japan), para obtenção das imagens. A infiltração marginal foi analisada por meio do software Image Tool (v.2.0; UTHSCSA, San Antonio, USA). A mensuração foi realizada, após a remoção do material retrobturador, avaliada desde a superfície apical da parede da cavidade retrógrada até a penetração máxima da solução corante.

Os resultados foram submetidos à análise estatística pelos testes ANOVA e teste de Tukey.

\section{Resultados}

$\mathrm{O}$ aspecto representativo da infiltração marginal observada no dentes bovino está representado na Figura 1. Os dados obtidos neste estudo foram submetidos ao teste ANOVA e teste de Tukey, onde se demonstrou menor infiltração apical para o Sealer $26(\mathrm{p}<0,05)$. O MTA e OZE apresentaram infiltração apical semelhante $(\mathrm{p}>0,05)$.

Os valores médios de infiltração marginal observados nos grupos experimentais de acordo com os resultados estatísticos estão expressos na Figura 2.

O grupo controle positivo apresentou infiltração marginal máxima, ou seja, completa infiltração em toda a parede da cavidade retrógrada. Já o grupo controle negativo apresentou infiltração zero, os espécimes não apresentaram infiltração do corante na cavidade retrógrada. 


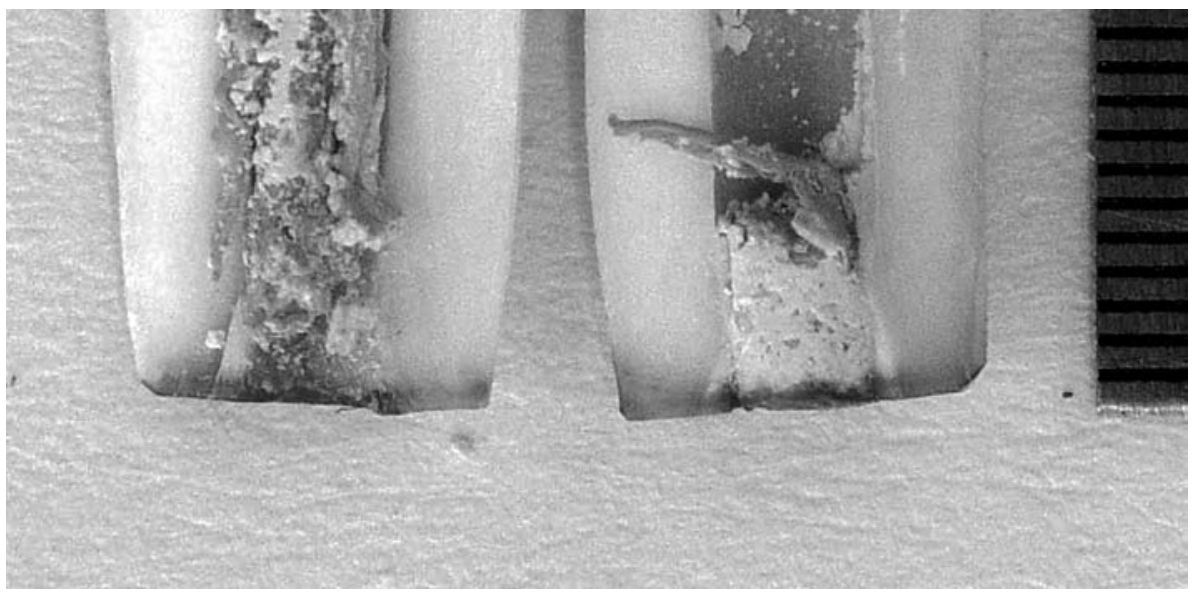

FIGURA 1 - Aspecto representativo da infiltração marginal observada após corte longitudinal do dentes bovino.

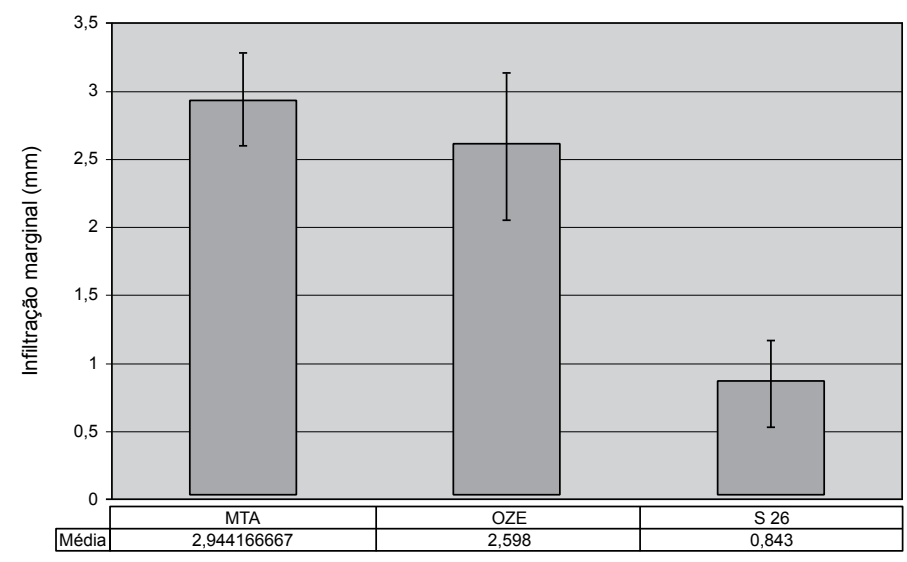

FIGURA 2 - Infiltração marginal média e desvio-padrão (em mm) observados para os grupos MTA, Óxido de Zinco e Eugenol (OZE) e Sealer 26 (S26).

\section{Dıscussão}

Para obtenção de sucesso nas obturações retrógradas, é fundamental o emprego de um material que apresente adequada capacidade seladora, minimizando as consequiências da infiltração marginal.

Estudos de infiltração marginal têm sido realizados com soluções corantes como Rodamina B, azul de metileno, entre outros marcadores. Entretanto o uso do corante azul de metileno tem sido questionado para avaliação da infiltração marginal. Kontakiotis et al. ${ }^{10}$ (1997), Wu et al. ${ }^{28}$ (1998), Moraes et al. ${ }^{12}$ (2000) consideram que esta solução corante sofre ação de descoloração na presença de substâncias alcalinas.
Desta forma, materiais alcalinos como o MTA não apresenta compatibilidade com a solução de azul de metileno. Duarte et al. ${ }^{6}$, em 2003 avaliaram dois materiais a base de MTA demonstrando o potencial de alcalinização do MTA, o qual proporciona aumento do $\mathrm{pH}$. Deste modo, a possibilidade de descoloração do corante azul de metileno em contato com o MTA contra-indica o seu uso (Silva-Neto et al. ${ }^{18}$, 2001, Tanomaru-Filho et al. ${ }^{20}$ 2005).

A solução de Rodamina B foi empregada neste estudo para avaliação da capacidade seladora, em função da avaliação de materiais alcalinos como o MTA, sendo também o corante indicado por Tanomaru-Filho et al. $^{21}(2005)$. 
Em nosso estudo, foram utilizados dentes bovinos, pois estes têm sido utilizados em estudos de infiltração marginal; testes de adesão de materiais restauradores; avaliação antimicrobiana de medicamentos intracanal e cimentos obturadores, conforme relatado por Nakamichi et al. ${ }^{13}$ (1983). Os dentes bovinos são morfologicamente similares aos dentes humanos, apresentam menor variabilidade de permeabilidade do tecido; anatomia interna com menor variação. A dentina radicular bovina apresenta túbulos dentinários, densidade e morfologia semelhantes à dentina humana (Nakamichi et al. ${ }^{13}$, 1983; Saunders ${ }^{15}$, 1988; Tagami et al. ${ }^{19}$, 1990; Reeves et al. ${ }^{14}, 1995$; Correa et al. $\left.{ }^{5}, 2003\right)$. A disponibilidade e facilidade de obtenção dos dentes bovinos quando comparado aos dentes de humanos, tornam o seu emprego com opção para as pesquisas in vitro.

A maioria dos trabalhos encontrados na literatura (Bouillaguet et al. ${ }^{3} 2003$; Burrow et al. ${ }^{4} 1996$; Kim et al. ${ }^{9} 2002$; Schilke et al. ${ }^{16}$ 2000) apontam a dentina de incisivos bovinos como substrato para substituição da dentina humana em trabalhos in vitro.

De acordo com os resultados obtidos neste estudo o MTA e o OZE demonstraram ser semelhantes entre si quanto à capacidade seladora apical, concordando com os resultados de Tanomaru-Filho et al. ${ }^{21}$, em 2005, empregando dentes de humanos. O Sealer 26 apresentou melhor selamento marginal que os demais materiais, sendo que estes resultados para o material em obturações retrógradas concordam com Tanomaru Filho et al..$^{22} 2006$. Em estudo em perfurações de furca, Silva-Neto et al. ${ }^{17}$ (2003), encontraram bons resultados para um material experimental a base de resina epóxica e hidróxido de cálcio, com resultados superiores ao Pro-Root MTA e MTA-Angelus utilizando como corante a Rodamina B.

Em outro experimento, Silva-Neto et al. ${ }^{18}$ (2001), utilizando o corante Rodamina B na avaliação de in- filtração, observaram que o MTA-Angelus apresentou capacidade seladora semelhante à do Super-EBA em obturações retrógradas. Este resultado é também concordante com os resultados obtidos neste estudo. Entretanto alguns estudos como os de Torabinejad et al. ${ }^{25}$ (1994), Aqrabawi et al. ${ }^{1}$ (2000), obtiveram resultados superiores para o MTA em retrobturações, porém, utilizando como marcador o corante azul de metileno.

Neste estudo, onde utilizamos dentes bovinos e o corante de Rodamina B, o MTA-Angelus apresentou resultado semelhante de selamento ao OZE. O Sealer 26 apresentou melhores resultados estatísticos, proporcionando selamento apical superior ao MTAAngelus e OZE. Em outro estudo de infiltração, mas com o corante azul de metileno Tanomaru-Filho et al. ${ }^{24}$ (2003), encontraram o Pro-Root MTA e MTA-Angelus com resultados semelhantes ao Sealer 26 e superiores ao OZE em obturações retrógradas. Esses resultados sugerem que a avaliação da capacidade seladora para materiais a base de MTA pode ser influenciada pela solução corante utilizada.

Os resultados obtidos neste estudo sugerem que o modelo experimental com dentes bovinos pode ser utilizado para avaliação da capacidade seladora de materiais utilizados em obturações retrógradas.

\section{Conclusão}

Baseado na metodologia empregada e nos resultados obtidos neste estudo podemos concluir que:

1) Houve menor infiltração apical para o Sealer $26(\mathrm{p}<0,05)$

2) O MTA e OZE apresentaram infiltração apical semelhante $(\mathrm{p}>0,05)$;

3) $\mathrm{O}$ modelo experimental com dentes bovinos foi compatível com a avaliação da infiltração apical em obturações retrógradas. 


\section{Abstract}

The sealing ability of materials used in endodontics is studied by dye leakage studies. The goal of the present study was to evaluate of the apical sealing ability of root-end filling materials using as experimental model the bovine teeth. Forty bovine incisors recently extracted, with straight roots were submitted to root canal instrumentation and obturation. After apical resection, retrograde cavities were prepared with retro-tips in ultrasonic device. Then, the teeth were randomly divided into four experimental $(n=12)$ and four control groups $(n=4)$. The materials used were: Group I-gray MTA Angelus; Group II - zinc oxide-eugenol sealer (ZOE); Group III - Sealer 26. Then, the teeth were immersed in 0,2\% rhodamine B solution under vacuous environment for 48 hours. After this period, the teeth were longitudinally sectioned, the images of the teeth were digitalized and leakage was evaluated using the program Image Tool. Positive and negative control groups presented maximum apical infiltration or zero, respectively. Results were submitted to statistical analysis (ANOVA and Tukey's test), demonstrating the least apical leakage for Sealer $26(p<0,05)$. MTA and OZE presented similar apical leakage ( $p>0,05)$. Sealer 26 provides greater apical sealing then MTA and OZE, and the experimental model with bovine teeth is compatible for the evaluation of the apical leakage in retrograde fillings.

\section{UNITERMS}

Endodontics; dental leakage; root retrofilling materialo.

\section{REFERÊNCIAS}

1. Aqrabawi J. Sealing ability of amalgam, super EBA cement, and MTA when used as retrograde filling materials. Br Dent J 2000 Mar.; 11(5):266-8.

2. Bernabé PFE. Estudo histológico realizado em dentes de cães com lesão periapical após apicectomia e tratamento endodôntico via retrógrada. Influência do nível da obturação e do material obturador. [tese de Livre-Docência] - Araçatuba: Faculdade de Odontologia de Araçatuba, Universidade Estadual Paulista-UNESP: 1994

3. Bouillaguet S, Troesch S, Wataha JC, Krejci I, Meyer JM, Pashley DH. Microtensile bond strength between adhesive cements and root canal dentin. Dent Mater. 2003 May.; 19(3):199-205.

4. Burrow MF, Sano H, Nakajima M, Harada N, Tagami J. Bond strength to crown and root dentin. Am J Dent 1996 Oct.; 9(5):223-9.

5. Correa MD, Anauate-Netto C, Youssef MN, Carmo ARP. Estudo micromorfológico comparativo entre dentina bovina e humana ao MEV. Rev Pos Grad 2003; 10(4):312-16.

6. Duarte MAH, Demarchi AC, Yamashita JC, Kuga MC, Fraga SC. pH and calcium ion release of 2 root-end filling materials. Oral Surg Oral Med Oral Pathol Oral Radiol Endod 2003 Mar.; 95(3):345-7.

7. Friedman S, Rotstein I, Koren L, Trope M. Dye leakage in retrofilled dog teeth and its correlation with radiographic healing. J Endod. 1991 Aug.; 17(8):392-5.

8. Holland R, Filho JA, De Souza V, Nery MJ, Bernabe PFE, Junior ED. Mineral trioxide aggregate repair of lateral root perforations. J Endod 2001 Apr.; 27(4):281-4.

9. Kim KH, Ong JL, Okuno O. The effect of filler loading and morphology on the mechanical properties of contemporary composites. J Prosthet Dent 2002 Jun.; 87(6):642-9.

10. Kontakiotis EG, Wu MK, Wesselink PR. Effect of calcium hydroxide dressing on seal of permanent root filling. Endod Dent Traumatol 1997 Dec.; 13(6):281-4.

11. Lee SJ, Monsef M, Torabinejad M. Sealing ability of a mineral trioxide aggregate for repair of lateral root perforations. J Endod 1993 Nov.; 19(11):541-4.

12. Moraes IG, Nunes E, Berbert A, Duarte MAH, Betti LV. Influência do hidróxido de cálcio e do EDTA na marcação da infiltração marginal de azul de metileno em obturação de canais radiculares. Rev FOB 2000 jan/jun.; 8(1):37-44.

13. Nakamichi I, Iwaku M, Fusayama T. Bovine teeth as possible substitutes in adhesion test. J Dent Res 1983 Oct.; 62(10):1076-81.

14. Reeves GW, Fitchie JG, Hembree JH Jr, Puckett AD. Microleakage of new dentin bonding systems using human and bovine teeth. Oper Dent 1995 Dec.; 20(6):230-5.

15. Saunders WP. The shear impact retentive strengths of four dentine bonding agents to human and bovine dentine. J Dent 1988 Oct.; 16(5):233-8.

16. Schilke R, Lisson JA, Bauss O, Geurtsen W. Comparison of the number and diameter of dentinal tubules in human and bovine dentine by scanning electron microscopic investigation. Arch Oral Biol 2000 May.; 45(5):355-61.

17. Silva Neto UX, Moraes, IG. Capacidade seladora proporcionada por alguns materiais quando utilizados em perfurações na região de furca de molares humanos extraídos. J Appl Oral Sci 2003 jan./mar.; 11(1):27-34

18. Silva Neto UX, Brochado VHD, Gonçalves-Júnior JF, Westphalen VPD, Moraes IG. Infiltração marginal em obturações retrógradas realizadas com ProRoot-MTA, MTA-Angelus e Super-EBA. J Bras Endod 2001; 4(13): 149-52.

19. Tagami J, Tao L, Pashley DH. Correlation among dentin depth permeability and bond strength of adhesive resins. Dent Mater 1990 Jan.; 6(1):45-50.

20. Tanomaru-Filho M, Figueiredo FA, Tanomaru, JMG. Effects of different dye solutions on the evaluation of the sealing ability of mineral trioxide aggregate. Bras Oral Res 2005 abr./jun.;19(2):119-22.

21. Tanomaru-Filho M, Jorge EG, Barbizam JVB, Tanomaru JMG. Avaliação da capacidade seladora apical de materiais retrobturadores à base de mineral trióxido agregado (MTA) e do óxido de zinco e eugenol, em corante Rodamina B. RFO UPF 2005 jul./dez.; 10(2):59-62.

22. Tanomaru-Filho M, Jorge EG, Tanomaru JMG. Avaliação da capacidade seladora apical de materiais retrobturadores empregando corante Rodamina B. J Bras Endod 2006; 6(24):89-93. 
23. Tanomaru-Filho M, Luis MR, Leonardo MR, Tanomaru JMG, Silva LAB. Evaluation of periapical repair following retrograde filling with different root-end filling materials in dog teeth with periapical lesions. Oral Surg Oral Med Oral Pathol Oral Radiol Endod 2006 Jul.; 102(7):127-32.

24. Tanomaru-Filho M, Tanomaru JMG, Ishikawa T.M. Capacidade de selamento apical de materiais retrobturadores à base de Agregado de Trióxido Mineral. J Bras Endod 2003; 4(12):20-3.

25. Torabinejad M, Higa RJ, McKendry DJ, Pitt, Ford TR. Dye leakage of four root end filling materials: effects of blood contamination. JEndod 1994 Apr.; 20(4):159-63.

26. Torabinejad M, Pitt Ford TR, McKendry DJ, Abedi HR, Miller DA, Kariyawasam SP. Histologic assessment of mineral trioxide aggregate as a root-end filling in monkeys. J Endod 1997 Apr.; 23(4):225-8.

27. Torabinejad M, Watson TF, Pitt Ford TR. Sealing ability of a mineral trioxide aggregate when used as a root end filling material. J Endod 1993 Dec.; 19(12):591-5.
28. Wu MK, Kontakiotis EG, Wesselink PR. Decoloration of $1 \%$ methylene blue solution in contact with dental filling materials. J Dent. 1998 Sep.; 26(7):585-9.

29. Wu MK, Kontakiotis EG, Wesselink PR. Long-term seal provided by some root-end filling materials. J Endod 1998 Aug.; 24(8):557-60.

Recebido em: 18/12/06 Aprovado em: 23/08/07

Endereço para Correspondência: Prof. Dr. Mário Tanomaru-Filho e-mail: tanomaru@uol.com.br Rua Humaitá, 1901, apto 182 - Centro CEP 14801-385 - Araraquara - SP Tel: (16)3301-6390 - (16) 9783-3054 